\title{
Análisis del componente social de la ganadería en una región al norte de Colombia (Municipio de Montería) ${ }^{1}$
}

\author{
Analysis of the Social Component of Livestock in a Northern \\ Region of Colombia (Municipality of Monteria)
}

Teonila Aguilar Jiménez ${ }^{2}$

Doris Serrano Amaya ${ }^{3}$

Para citar este artículo, utilice el nombre completo así:

Aguilar, T. \& Serrano, D. (2015). Análisis del componente social de la ganadería en una región al norte de Colombia (Municipio de Montería). Perspectiva Geográfica, 20(1), 133-154.

\section{Resumen}

El presente artículo analiza las características sociales de los ganaderos del municipio de Montería; se tienen en cuenta variables como el nivel educativo, edad, género, formas de tenencia de la tierra, mano de obra y fuente de ingresos de los productores, con el fin de aportar elementos de análisis que permitan entender su relación con el atraso del sector, ya que son ellos quienes dirigen e implementan el conjunto de prácticas que determina el sistema productivo predominante. Para la recolección de la información, se aplicaron 242 encuestas, a igual número de ganaderos, distribuidas en

1 Este trabajo se deriva de la tesis de maestría, intitulada: "Caracterización social, tecnológica y ambiental de la ganadería en el municipio de Montería. Un análisis desde la geografía". Grupo de investigación: Geografía y Ambiente Universidad de Córdoba.

2 Licenciada en Ciencias Sociales, MsC en Geografía. teonila7@gmail.com

3 Agróloga, Especialista en SIG, MsC en Geomática. dserranoamaya@gmail.com 
las nueve zonas o Unidades Espaciales de Funcionamiento, UEF, en las que se divide el área rural del municipio de Montería. Los resultados del estudio muestran que existe una alta proporción de ganaderos con bajos niveles de estudio y avanzada edad, con predominio de propietarios varones y en donde prevalece la pequeña propiedad.

Palabras clave: características sociales, edad, finca ganadera, mano de obra, nivel educativo, tenencia de tierra.

\begin{abstract}
This article analyzes the social characteristics of the cattle-raisers of the municipality of Monteria, taking into account variables such as education level, age, gender, forms of land tenure, labor and source of income for producers, in order to provide elements of analysis to understand their relationship with the backwardness of the sector, since it is they who lead and implement a set of practices that determines the predominant production system. To collect information 242 surveys were applied to the same number of cattle-raisers spread across nine areas or Operating Units Space - OUS, in which the rural area of the municipality of Monteria is divided. The study results show that there is a high proportion of cattle-raisers with low levels of study and advanced age with predominance of male owners and where small property prevails.
\end{abstract}

Keywords: social characteristics, age, cattle farm, labor land, education, ownership. 


\section{Introducción}

En Montería, la ganadería es una actividad de gran importancia económica; su inventario bovino se destaca como el más grande del departamento con, aproximadamente, unas 427.996 cabezas, distribuidas en 3.365 fincas; a su vez, el departamento de Córdoba ocupa el cuarto puesto, en el ámbito nacional, después de Meta, Casanare y Antioquia, con unas 2.419.559 cabezas; en el ámbito local, ocupa el primer puesto, siguiéndole en importancia Cesar (DANE, 2010). Lo anterior, demuestra la relevancia de este sector en la economía local y regional; sin embargo, es duramente criticado por su carácter extensivo, baja productividad y altos costos ambientales atribuidos, generalmente, a un bajo nivel de tecnificación.

La ganadería es una actividad con una gran heterogeneidad de componentes; un complejo sistema que presenta diversas formas de organización técnica, económica y social. A pesar de tal heterogeneidad, la mayoría de las investigaciones realizadas en el área de estudio se han desarrollado desde la especialidad zootécnica y abundan temas como sistemas de producción, sa- nidad animal y métodos de alimentación; sin duda, de gran valor en su campo. Sin embargo, no se le ha dado el mismo tratamiento al estudio del componente social; este es un aspecto importante dentro de las explotaciones ganaderas, pues en estas no solo se ponen en práctica un conjunto, más o menos complejo, de medios de producción, sino que estos son el resultado de una serie de decisiones tomadas por personas con objetivos y finalidades determinadas; decisiones en las que influyen las características personales y sociales de quien las toma (Serrano \& Mantecón, 2003).

De acuerdo con lo anterior, se debe tener en cuenta que la ganadería se desarrolla bajo la orientación de un productor; es decir, el ganadero es quien toma decisiones en su finca y es ahí en donde el componente social cobra relevancia, ya que atributos como el nivel educativo, la edad, el género, forma de tenencia de la tierra, tamaño de los predios y fuente de los ingresos pueden determinar las relaciones sociales, económicas y productivas dentro de la actividad ganadera. Por tanto, se busca asociar cómo estas características se relacionan cuando se adoptan procesos tecnológicos en el interior de las fincas. Este aspec- 
to ha sido explicado por Rodgers (1962), en su teoría de la difusión de innovaciones, en la cual, entre otros factores, plantea que para analizar la adopción y difusión de procesos tecnológicos es necesario el conocimiento de las características sociales que distinguen a los individuos o grupos dentro de un sistema social, y estas características pueden facilitar o impedir la adopción y difusión de elementos nuevos en una estructura social.

En esta misma línea, Hagerstrand (1967), en su modelo de difusión de innovaciones, establece que una innovación son todas aquellas ideas, prácticas u objetos que se perciben como nuevos, y cuando se utilizan mejoran los resultados. Tales innovaciones se difunden por el espacio y el tiempo a través de unos canales que limitan o alteran su normal desarrollo. Los limitantes son de diversos tipos; van desde los inherentes a las innovaciones mismas hasta aquellas que se relacionan con las características sociales del adoptante. Hagerstrand (1967), registra que 1os niveles de resistencia para adoptar elementos nuevos difieren entre las personas; ese hecho es el resultado de considerar las características personales de cada individuo junto con las del grupo del que forma parte (Tullas, 1983). Características que terminan ejerciendo influencia sobre los resultados de los sistemas de producción empleados en las fincas ganaderas.

Para un ganadero, una innovación puede ser una nueva variedad de pasto que prometa mejores rendimientos y se adapte mejor a períodos de sequías o de mucha humedad, la introducción de alternativas nutricionales que puedan representar economía y mayores ganancias; utilización de nuevas razas que aseguren mayores rendimientos, de acuerdo con la dedicación a determinados ciclos productivos, nuevas técnicas para el manejo y conservación de praderas, entre muchos otros ejemplos que pueden relacionarse a partir de la ganadería. Cada uno de estos procesos, si se implementan, significan mejores rendimientos y hasta sostenibilidad de la producción, pero se pueden truncar si son afectados por las características particulares de quienes las adoptan; en este caso, de los ganaderos.

Por tal razón, el presente estudio se propone identificar las características sociales de los productores, comparando su comportamiento 
en las diferentes zonas del municipio, con el objeto de ponerlas en evidencia y analizar cómo pueden contribuir a uno de los principales problemas que enfrenta el sector: el bajo nivel de tecnificación, y se puedan poner en marcha estrategias de mejoramiento.

\section{2. Área de estudio}

El municipio de Montería tiene una extensión territorial de 320.459,7 hectáreas; de estas 4.092,0 hectá- reas corresponden a la superficie urbana, representando al 1,3\% del total municipal; mientras que el área rural abarca unas $316.367,7$ hectáreas, lo que equivale al 98,7\% del territorio. Administrativamente, el área rural del municipio está conformada por 29 corregimientos, en los que existen 168 veredas, distribuidas en 9 Unidades Espaciales de Funcionamiento, UEF (Alcaldía de Montería, 2002) (Figura 1).

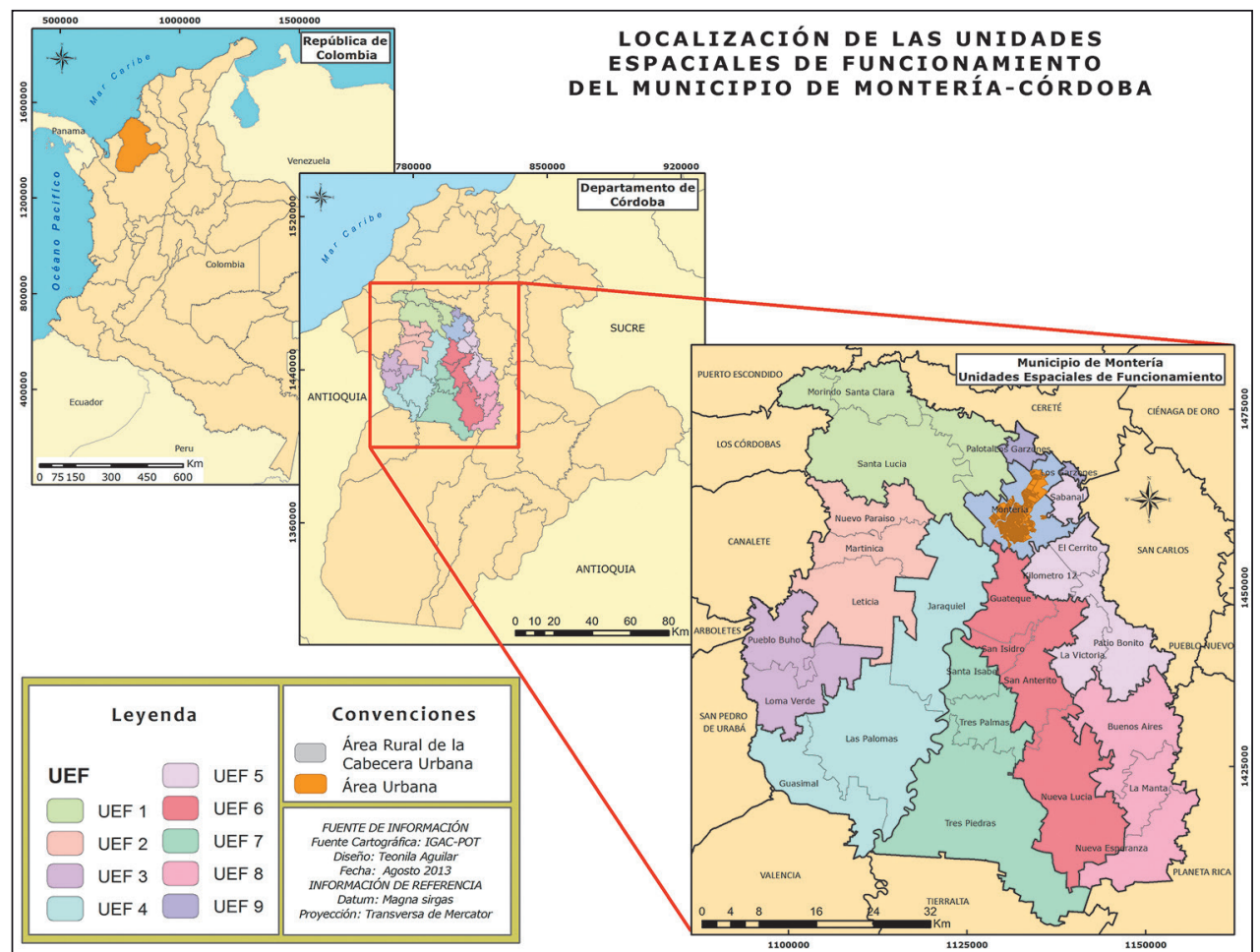

Figura 1. Mapa de localización del área de estudio

Fuente: Cartográfica IGAC, 2008; POT Municipio de Montería, 2002-2015. 
Topográficamente, se caracteriza por ser, en su gran mayoría, suelos de planicie, con algunas elevaciones menores, derivadas de las serranías de Abibe al occidente y San Jerónimo al oriente. Es recorrido por su principal vértebra hidrográfica, el río Sinú, configurando el valle del mismo nombre, considerado uno de los más fértiles del mundo. Además, la presencia de varios cuerpos de agua, riachuelos y quebradas genera importantes beneficios en sus zonas de influencia. Estas características lo hacen una zona propicia para el desarrollo agropecuario.

\section{Materiales y métodos}

Para la recolección de la información, se aplicó una encuesta semiestructurada, a una muestra de 242 fincas ganaderas. Para la determinación de la muestra, se tuvo en cuenta el tamaño de la población: 2.642 fincas ganaderas (se desestimó algunas fincas ubicadas en zonas de difícil acceso y problemas de inseguridad), y su distribución en las nueve zonas o Unidades Espaciales de Funcionamiento, UEF, en las que se divide el área rural del municipio de Montería; el tamaño de la muestra se determinó en 242 fincas, con un nivel de significancia del $95 \%$, y un error máximo permisible del $6 \%$.

El tamaño de la muestra es ahora dividido en estratos, determinados por las zonas o Unidades Espaciales de Funcionamiento, UEF, las veredas que las integran y los predios o fincas ganaderas.

La selección del número de veredas por UEF, se prefijó en un 30\%, considerando que estas daban una buena representación del total de predios en cada UEF. Luego, se determinó el número total de fincas ganaderas en cada vereda y se seleccionaron, al azar, las fincas por encuestar. El número de fincas seleccionadas, se determinó de manera proporcional con el número total de predios de cada vereda; así, se garantizó la muestra de 242 fincas.

\section{Resultados y discusión}

El componente social de los ganaderos está integrado por atributos diferenciadores, como el género, la edad, el nivel educativo, tamaño de los predios, tenencia de la tierra y fuente de los ingresos económicos; características que juegan un papel importante, dada su influencia so- 
bre los procesos de toma de decisiones.

\subsection{Nivel educativo y ganadería}

El papel de la educación en el desarrollo, y su incidencia en los roles que cumplen los actores en las diferentes actividades productivas, ha sido destacado en varias investigaciones de organismos internacionales y nacionales, pues permite que las personas alcancen una mejor calidad de vida; es una de las herramientas con que cuenta la sociedad para transformar su realidad (Banco Mundial, 2007; Salgado y Tréllez, 2009; FAO, 2011). La educación permite que las personas amplíen sus perspectivas de análisis, los beneficios o perjuicios que pueden traer determinadas actividades; facilita la adopción de tecnologías al comprender su importancia en el crecimiento de las actividades productivas.

De acuerdo con los resultados, en el área de estudio se encuentra que el $38 \%$ de los ganaderos alcanzaron solo estudios primarios. Este resultado no es homogéneo en todo el municipio, porque en algunas zonas el problema es más agudo; es el caso de las unidades 3,4 y 1 ; en donde, en el mismo orden, el $71,4 \%, 62,1 \%$ y $48,6 \%$ de los ganaderos establecidos allí, solo terminaron ese ciclo de estudio. Las zonas 3 y 4 están ubicadas espacialmente en la parte suroccidental, hacia la margen izquierda del río Sinú, bordeadas por las estribaciones de la serranía de Abibe; limitan con los municipios de Tierralta, Valencia y San Pedro de Urabá, en donde, históricamente, se ha presentado conflictos por el dominio del territorio y son regiones con necesidades básicas insatisfechas. Estas características están presentes también en algunas zonas periféricas del municipio de Montería, coincidiendo con la ubicación de las unidades espaciales en donde el nivel educativo de los ganaderos es más bajo; esto quiere decir que los ganaderos con el más bajo nivel educativo se ubican en las zonas periféricas del municipio.

En contraste, los mayores porcentajes de ganaderos con formación profesional se encuentra, en su orden, en las unidades 9, 7, 6 y 5, en donde alcanzan porcentajes de $50 \%, 40,7 \%, 40 \%$ y $30,5 \%$ (Figura $2)$. Las unidades con mayor porcentaje de profesionales se ubican en la margen derecha y a lo largo del río. Se debe tener en cuenta que los suelos de estas áreas son 


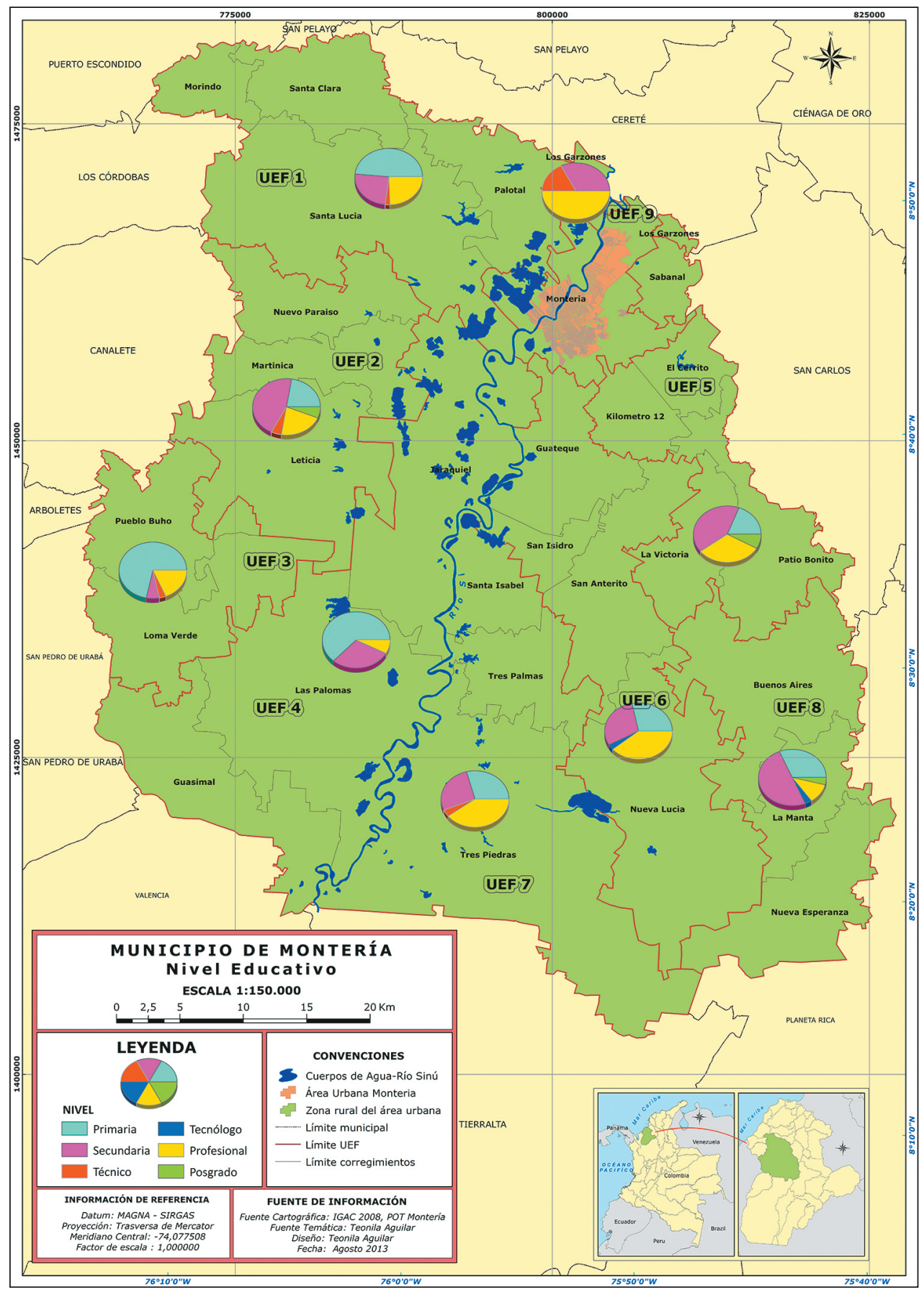

Figura 2. Nivel educativo de los ganaderos.

Fuente: Elaboración propia, con datos recolectados en campo. 
de buena calidad; la mayoría de origen aluvial, en donde el río ejerce una vasta influencia. También, allí se ha ido construyendo unos equipamientos estratégicos para la comercialización y sacrificio de ganado. Además, esta parte limita con el municipio de Planeta Rica, el cual se ha venido consolidando como un centro de acopio lechero reconocido en el departamento; sin dejar de registrar la importante dinámica que ejerce, en esta parte, la vía que comunica el departamento de Córdoba con centros de comercialización de ganado en el interior del país. Cuando las personas son instruidas se les facilita entender, analizar y sacar provecho de ciertas ventajas que el ambiente físico y una localización estratégica pueden proveerles.

Estos resultados presentan cierta similitud con estudios realizados en España, en donde se encuentra una proporcionalidad muy alta de ganaderos sin estudios y con estudios primarios (Morantes, et al., 2014).

\subsection{Edad de los ganaderos}

La edad promedio de los ganaderos de todo el municipio es de 56 años; se presenta una mayor frecuencia en las edades entre 50 y 60 años, con un $33.33 \%$, seguido de los rangos de edad 40-50 y 60-70 años, con $27.00 \%$ y $20.67 \%$, respectivamente.

Espacialmente, se encuentra algunas diferencias en el comportamiento de esta variable. Por ejemplo, en las zonas 1, 2, 3 y 5 los ganaderos mayores de 65 años representan porcentajes entre el $26 \%$ y el 29\%. En las zonas 6, 7, 8 y 9 esos porcentajes constituyen entre el $30 \%$ y el $35.5 \%$; es la unidad 8 , la que tiene el más alto porcentaje de ganaderos mayores de 65 años. Los datos, en general, arrojan un nivel alto de envejecimiento en los ganaderos del municipio, teniendo en cuenta que la tasa de envejecimiento del país, en el último censo, alcanzó apenas el 6,3\%. Este fenómeno responde a una tendencia mundial que afecta a todos los países, producto de la transición demográfica que han atravesado las regiones a lo largo del tiempo y que afecta de manera especial a las zonas rurales, por el efecto migratorio de los jóvenes hacia los centros urbanos.

Algunos estudios demuestran que la edad de los productores puede convertirse en una característica 
perturbadora de los procesos de modernización tecnológica; son los jóvenes más propensos a implementar innovaciones (Velasco, et al., 2009 y Morantes, et al., 2014). Al mismo tiempo, esos procesos son más reticentes en grupos poblacionales de edad mayor. Además, la capacidad productiva también disminuye con el paso de los años. Las personas de edad avanzada vienen de una época en la que no existían muchas de las tecnologías empleadas hoy; eso, complementado con el nivel educativo que ostentan, puede dificultar la modernización de este sector.

\subsection{Género}

Las asimetrías de género, resultantes de la comparación de su participación en las diferentes actividades del sector agropecuario, aumentan, disminuyen o se igualan dependiendo del tipo de actividad. Para el caso de la ganadería, en particular, la brecha se afianza cuando se trata de la producción de ganado mayor. En el municipio de Montería se encuentra que un $80,1 \%$ de los propietarios de las fincas ganaderas encuestadas son hombres, y un $16,5 \%$, son mujeres. El comportamiento no es homogéneo en toda la región, ya que se encuentran zonas en donde el porcentaje de mujeres ganaderas supera el promedio del municipio. Es el caso de la unidad 9, en donde un $33,3 \%$ de los productores son mujeres, seguida de la unidad 6 , con un $23,5 \%$, y la unidad 1 , con un $21,1 \%$. Los resultados tienen similitud con investigaciones internacionales, en donde resulta escasa la participación femenina en las actividades ganaderas, especialmente, en la producción de bovinos (Banco Mundial, 2010; Vilaboa-Arroniz, et al., 2009). Existe una tendencia cultural, derivada de la tradicional división sexual del trabajo, en donde el ganadero varón ha ejercido como principal actor en esta actividad. Algunas investigaciones sostienen que la baja participación de las mujeres en la ganadería bovina se relaciona con las desventajas que ellas tienen frente a los hombres para acceder a la tierra. En el ámbito mundial, menos del $20 \%$ de los propietarios de tierras son mujeres, situación que cobra mayor relevancia en el mundo en desarrollo (FAO, 2010).

Los porcentajes más altos de mujeres ganaderas se encontraron en las zonas 9, 6 y 1; esta ubicación estaría determinada por una mayor 
cercanía de esas zonas con la cabecera municipal. Toda la unidad 9 tiene contacto con el perímetro urbano de la ciudad de Montería $\mathrm{y}$, aunque las unidades 1 y 6 no limitan directamente con el casco urbano, varias de sus veredas se encuentran a distancias cortas de la cabecera, con mejores condiciones de seguridad y de acceso vial. En contraste, las unidades que presentan los porcentajes más altos de hombres propietarios son, precisamente, aquellas cuyas veredas se encuentran más retiradas de la cabecera municipal, caso de la unidad 7 y 8; existe, así, una relación entre distancia y ubicación de mujeres al frente de explotaciones ganaderas.

A pesar de que en el país no existen estudios que permitan comparar, en lo nacional, las diferencias regionales sobre la participación femenina en la ganadería, es importante tener en cuenta este componente, pues dos tercios de los más de 600 millones de ganaderos pobres en el mundo son mujeres y las investigaciones realizadas sobre el tema son pocas, al igual que los programas orientados a ese sector de la población (Njuki \& Sanginga, 2013; Kristjanson, et al., 2010).

\subsection{Fuentes de ingreso de los ga- naderos}

La dedicación exclusiva a la actividad ganadera por parte de los productores puede resultar benéfica para el desarrollo del sector, debido a que siempre se buscará potenciar los rendimientos en la búsqueda de mejores ganancias que permitan suplir los costos de sostenimiento de la finca y suplir las necesidades del grupo familiar del propietario. Sin embargo, hay quienes opinan que, en varios casos, cuando la ganadería se desarrolla solo de tiempo parcial y que el propietario recibe ingresos de otras actividades, esos recursos le permiten financiar inversiones en las propias explotaciones o superar períodos de crisis (Malaquín, 2009).

La ganadería es la única fuente de ingresos para la gran mayoría de los productores en seis de las nueve zonas del municipio de Montería; esto es, en las unidades $2,4,5$, 6,7 y 8 , con porcentajes que oscilan entre el $50 \%$ y el $80 \%$; son las unidades 5, 6 y 8 las que concentran el mayor número de ganaderos en esta condición (Tabla 1). Estas tres zonas son contiguas y vecinas entre sí y se ubican en la margen derecha del río, en el área de in- 
fluencia de los centros ganaderos locales de comercialización, lo que permite inferir que los productores dedicados mayoritariamente a sus explotaciones ganaderas tienden a aprovechar mejor todos los beneficios que el entorno les provea.

La ganadería también se practica en asocio con otras actividades, principalmente, con la agricultura. Las áreas con mayor porcentaje de ganaderos-agricultores se ubican en las unidades 3,4 y 1 , con porcentajes de $55 \%, 46,4 \%$ y 39,5 en el mismo orden. Estos ganaderos se ubican en la margen izquierda del río. Así, se encuentra que son, precisamente, esas mismas unidades en donde se concentran los predios de menor tamaño y en donde se ubican los más altos porcentajes de ganaderos con menor nivel educativo. Quiere decir, entonces, que los ganaderos poseedores de predios de menor tamaño (menores de 50 hectáreas) complementan la actividad con la agricultura y se ubican en las zonas periféricas del municipio, caso de las unidades 3 y 4 .

Tabla 1. Fuente de ingresos de los ganaderos

\begin{tabular}{|c|c|c|c|c|c|c|c|c|c|c|}
\hline \multirow{2}{*}{$\begin{array}{l}\text { FUENTES DE } \\
\text { INGRESO }\end{array}$} & & \multicolumn{9}{|c|}{ UNIDADES ESPACIALES DE FUNCIONAMIENTO } \\
\hline & & 1 & 2 & 3 & 4 & 5 & 6 & 7 & 8 & 9 \\
\hline \multirow[t]{2}{*}{$\begin{array}{l}\text { Solamente de } \\
\text { la Ganadería }\end{array}$} & Total & 12 & 10 & 9 & 15 & 24 & 20 & 13 & 24 & 2 \\
\hline & $\%$ & 31.6 & 52.6 & 31.0 & 53.6 & 85.7 & 60.6 & 50.0 & 80.0 & 33.3 \\
\hline \multirow[t]{2}{*}{$\begin{array}{c}\text { Ganadería y } \\
\text { agricultura }\end{array}$} & Total & 15 & 4 & 16 & 13 & 0 & 4 & 3 & 4 & 1 \\
\hline & $\%$ & 39.5 & 21.1 & 55.2 & 46.4 & .0 & 12.1 & 11.5 & 13.3 & 16.7 \\
\hline \multirow{2}{*}{$\begin{array}{l}\text { Ganadería y } \\
\text { profesión }\end{array}$} & Total & 11 & 5 & 3 & 0 & 4 & 9 & 10 & 2 & 3 \\
\hline & $\%$ & 28.9 & 26.3 & 10.3 & .0 & 14.3 & 27.3 & 38.5 & 6.7 & 50.0 \\
\hline \multirow[t]{2}{*}{ Otra } & Total & 0 & 0 & 1 & 0 & 0 & 0 & 0 & 0 & 0 \\
\hline & $\%$ & .0 & .0 & 3.4 & .0 & .0 & .0 & .0 & .0 & .0 \\
\hline
\end{tabular}

Fuente: Elaboración propia, a partir de la información recopilada en campo.

\subsection{Formas de tenencia y tamaño de las fincas}

El modelo de explotación ganadera reposa sobre una base productiva que es la propiedad de la tierra.
Los ganaderos propietarios de sus predios representan un 94,54\%. Solo el 5,46\% son arrendatarios, tendencia que se manifiesta en todas las zonas del municipio. 
Este es un aspecto positivo, ya que a la hora de implementar programas de asistencia estatal o gremial se parte de un elemento fundamental en la estructura agraria, como es la forma de tenencia de la tierra; en este caso, la propiedad. Esta situación representa una ventaja cuando se contrapone con la agricultura, otro renglón económico importante en el municipio, en donde todavía prevalece el arrendamiento de tierras como base de la estructura productiva de los pequeños productores de ese sector.

Para analizar los tamaños de la propiedad se sigue la clasificación realizada por el IGAC, en donde se define, como pequeña, la propiedad que tiene hasta 50 hectáreas; mediana, entre 50 y 500, y grande, más de 500 (Machado, 2011). Al analizar el tamaño de los predios ganaderos, se tiene que estos varían poco de una unidad espacial a otra; en la mayoría de las zonas, los predios que predominan son los menores de 50 hectáreas; solo en las unidades 2 y 5 la mayor proporción se encuentra en el rango de 50-500 hectáreas; en la unidad 9, el $100 \%$ de los predios tiene una extensión menor de 50 hectáreas
(Figura 3). El mayor fraccionamiento de la propiedad en la zona 9 está influenciado por el valor productivo de estas tierras, por su ubicación muy cerca de la cabecera municipal y por la alta demanda de suelos para construcción de viviendas campestres; aspectos que favorecen la desconcentración de la tierra en esta parte del municipio.

De la información anterior se colige que el tamaño de las fincas ganaderas sigue manteniendo la misma distribución y comportamiento que en el pasado, pues algunos estudios señalan que hacia los años 80 del siglo XX, la propiedad de la tierra tendía a orientarse hacia la disolución del latifundio en las áreas centrales y que esa forma de propiedad se limitaba a las áreas marginales, lo cual indicaría que esta modalidad de apropiación de la tierra se mantenía solo en aquellas áreas de escaso valor productivo (Montaña, 2002). De acuerdo con los resultados arrojados por este estudio, esa tradición se mantiene, pues en el municipio se da una tendencia a la desconcentración en las áreas donde la tierra es más productiva y está más valorizada. 


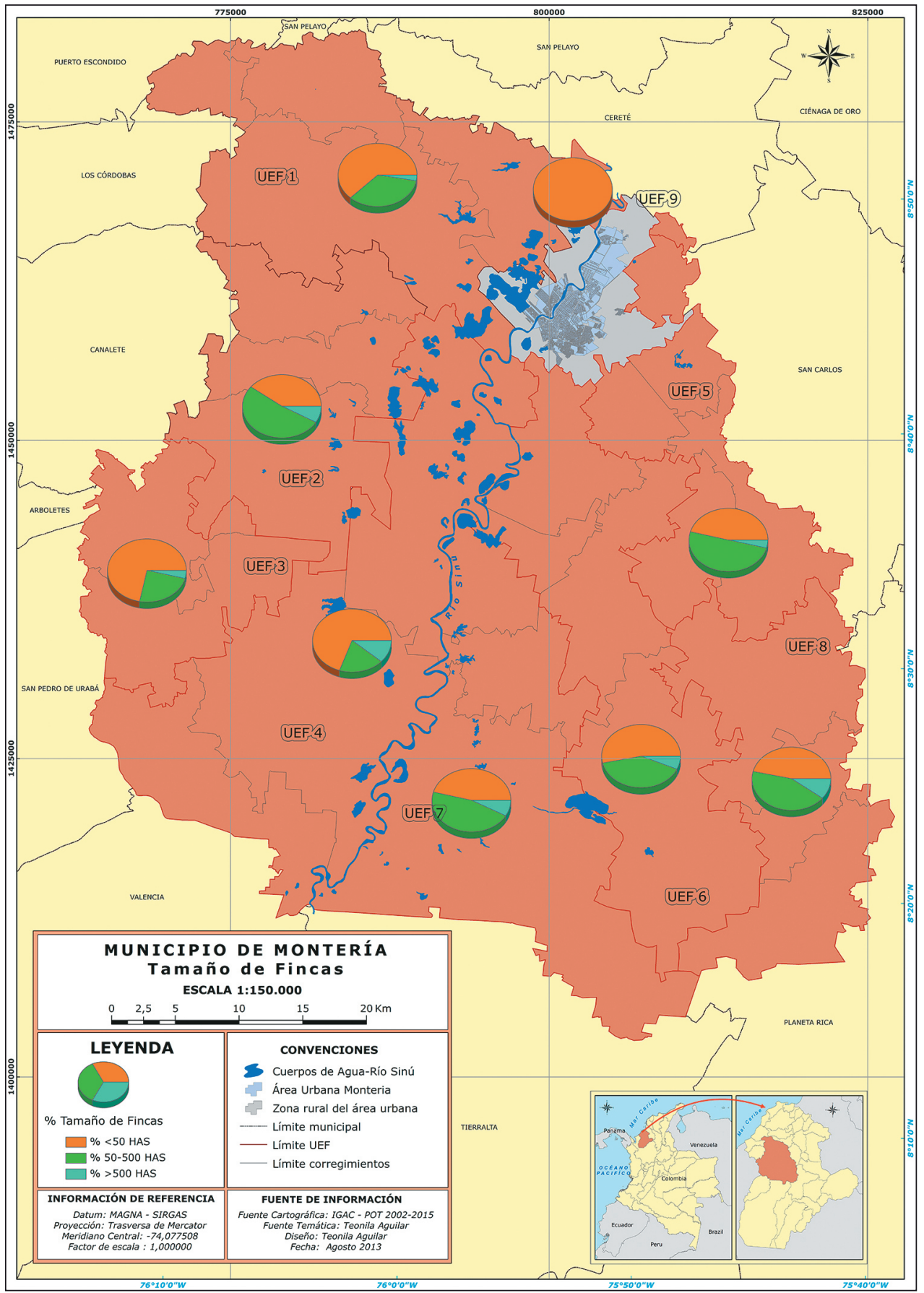

Figura 3. Mapa de tamaño de las fincas.

Fuente: Elaboración propia con información recopilada en campo. 
No obstante, lo anterior, es importante destacar que se sigue dando una proporción menor de propietarios que tiene bajo su dominio predios de mayor extensión, no siempre coincidentes con tierras marginales.

\section{Mano de obra}

El espacio rural del municipio de Montería se encuentra estructurado, sustancialmente, por la ganadería, como principal actividad económica. Sin embargo, esta importancia no se encuentra determinada por la vinculación de mano de obra, aspecto importante de la caracterización social de los productores. Se observa un alto porcentaje de fincas ganaderas que se sostienen con mano de obra familiar, con uno o dos trabajadores familiares no asalariados; es el caso de la unidad 4 con $72,7 \%$, la unidad 1 , con un $71,1 \%$, la unidad 2 , con $57,9 \%$, como las más destacadas.

Esto evidencia que, efectivamente, la contratación de mano de obra en los sistemas de producción ganadera del municipio es muy baja $\mathrm{y}$ explica también el estado de postración en que se encuentra la población rural municipal. Es la ganadería la actividad que ocupa gran parte de la tierra productiva y no genera el empleo necesario que ayude a contribuir con el mejoramiento de las condiciones de vida de los habitantes del entorno.

En cuanto la vinculación de la mano de obra asalariada permanente se encuentra porcentajes de fincas que no contratan ningún trabajador. Tales porcentajes oscilan entre un $10 \%$ y $30,3 \%$. Los más altos se encuentran en las fincas que contratan entre uno o dos trabajadores. En adelante, son muy pocas las fincas que contratan más de siete trabajadores permanentes durante el año. Por último, de las 27.908 hectáreas que abarcan las 242 fincas encuestadas, se encuentra 590 trabajadores permanentes, es decir, un trabajador para $47 \mathrm{hec}-$ táreas, aproximadamente.

Una de las mayores críticas que enfrenta la ganadería es su fracaso como modelo económico, ya que, siendo la actividad productiva más importante en la región, no ha podido jalonar su desarrollo. Una de sus grandes debilidades en este aspecto tiene que ver con la baja capacidad de absorción de mano de obra. Los resultados arrojan que se sigue sosteniendo, en gran parte, con mano de obra familiar; 
son coincidentes los resultados con otras investigaciones realizadas en el contexto latinoamericano (Holguín, et al., 2008).

\section{Relación entre el componente social y el componente tecnológico}

Analizar las características sociales es importante porque permite establecer ciertas relaciones entre estas y algunos aspectos del componente tecnológico. Una explotación ganadera tecnificada tiende a buscar las mejores condiciones del ganado, de tal manera que le permita obtener buenos rendimientos productivos; por tanto, se consideran variables de mucha importancia para analizar el componente tecnológico, la asistencia técnica para la salud animal, la alimentación y la infraestructura (Marín, 1996; Arias, 1997). Adicionalmente, Estrada (2002) resalta la importancia que juega la alimentación en este componente; destaca que los pastos son el principal recurso alimenticio, e indica que es la variable más crítica para definir la evolución de la producción y la productividad ganadera. En cuanto la infraestructura, su análisis resulta irrelevante en el municipio, ya que la inmensa mayoría de las fincas carecen de equipamientos técnicos especializados.

De esta manera, cuando se relaciona el nivel educativo y la implementación de una variable clave dentro de los procesos tecnológicos, como es la asistencia técnica para atender la salud de los bovinos, se encuentra que los porcentajes más altos de ganaderos que contratan este servicio son los profesionales. De los 65 ganaderos con formación superior, 21 contratan asesoría permanente y 23 de forma ocasional, lo cual representa un porcentaje del 8,7 y 9,5, respectivamente. En contraste, los que solo hicieron hasta la primaria contratan asesoría para la salud animal solo en un $1,6 \%$, de forma permanente y un $4.9 \%$ lo hace ocasionalmente (Tabla 2).

Los resultados contrastan con los encontrados en investigaciones similares, en donde la formación profesional de los ganaderos alcanza porcentajes superiores al 40\% (Morantes, et al., 2014), pero concluyentes en determinar que la educación es una variable significativa que permite medir la capacidad innovadora y gerencial de los productores ganaderos (Wosniak, 1984; Velasco, et al., 2009). 
En la zona de estudio, estas apreciaciones se pueden verificar al analizar la margen derecha del municipio, pues es allí donde se ubica las unidades espaciales que concentran el mayor porcentaje de ganaderos con formación profesional, el mayor porcentaje de fincas con dedicación completa a la actividad ganadera, el mayor porcentaje de fincas mejor acompañadas técnicamente y las zonas donde predominan los pastos mejorados, como la opción alimenticia preferida por los productores; destácase su presencia, especialmente, en las unidades 9, 8, 6, 5 y 4 . Todos estos aspectos evidencian la relación existente entre el nivel educativo de los ganaderos y su influencia a la hora de implementar procesos tecnológicos. Por consiguiente, es una variable que debe tenerse en cuenta a la hora de poner en marcha programas y políticas para el mejoramiento del sector.

Tabla 2. Nivel y educativo y asistencia técnica para la salud animal

\begin{tabular}{|c|c|c|c|c|c|c|}
\hline $\begin{array}{c}\text { Nivel } \\
\text { educativo }\end{array}$ & No. & $\%$ & Elemento tecnológico & Decisión & No. & $\%$ \\
\hline \multirow{3}{*}{ Primaria } & \multirow{3}{*}{93} & \multirow{3}{*}{38,4} & \multirow{3}{*}{$\begin{array}{l}\text { Asistencia técnica para } \\
\text { atender la salud animal }\end{array}$} & No tiene & 76 & 31,4 \\
\hline & & & & Ocasional & 12 & 4,9 \\
\hline & & & & Permanente & 4 & 1,6 \\
\hline \multirow{3}{*}{ Secundaria } & \multirow{3}{*}{76} & \multirow{3}{*}{31,4} & \multirow{3}{*}{$\begin{array}{l}\text { Asistencia técnica para } \\
\text { atender la salud animal }\end{array}$} & No tiene & 44 & 18,2 \\
\hline & & & & Ocasional & 24 & 9,9 \\
\hline & & & & Permanente & 7 & 2,9 \\
\hline \multirow{3}{*}{$\begin{array}{l}\text { Técnicos y } \\
\text { tecnólogos }\end{array}$} & \multirow{3}{*}{8} & \multirow{3}{*}{3,3} & \multirow{3}{*}{$\begin{array}{l}\text { Asistencia técnica para } \\
\text { atender la salud animal. }\end{array}$} & No tiene & 3 & 1,2 \\
\hline & & & & Ocasional & 4 & 1,6 \\
\hline & & & & Permanente & 1 & 0,4 \\
\hline \multirow{3}{*}{$\begin{array}{l}\text { Profesionales y } \\
\text { posgraduados }\end{array}$} & \multirow{3}{*}{65} & \multirow{3}{*}{26,4} & \multirow{3}{*}{$\begin{array}{l}\text { Asistencia técnica para } \\
\text { atender la salud animal }\end{array}$} & No tiene & 19 & 7,8 \\
\hline & & & & Ocasional & 23 & 9,5 \\
\hline & & & & Permanente & 21 & 8,7 \\
\hline
\end{tabular}

Fuente: Elaboración propia con información recopilada en campo.

Por otro lado, también se encuentra relación entre la edad avanzada y una menor tendencia a implementar procesos tecnológicos; por ejemplo, de los 55 ganaderos ma- yores de 65 años encuestados, solo el $16,3 \%$ contrata asesoría técnica para la salud animal de manera permanente, coincidente, de estos productores, el que ostentan nivel 
de formación de técnico a superior. El $45,4 \%$ de estos productores no contratan este servicio.

En ese mismo grupo de ganaderos, la mayor área en pastos mejorados que poseen representa $58,2 \%$, teniendo en cuenta que un $34,4 \%$ de estos productores tiene un nivel educativo de técnico a superior. El $29 \%$ posee la mayor área de su finca sembrada en pastos naturales, coincidente también con ganaderos que solo recibieron formación primaria.

Los ganaderos menores de 40 años representan el 8,67 \% en todo el municipio. De estos, el 14,3\% contrata asesoría técnica permanente, de los cuales el $52,4 \%$ tiene formación de técnica a superior y el $23,8 \%$ tiene formación primaria. En este mismo grupo de ganaderos menores de 40 años de edad utiliza pasturas mejoradas el $61,9 \%$ para la nutrición de sus animales.

De acuerdo con lo explicitado, la edad influye en la decisión de implementar elementos tecnológicos en las fincas de los productores, pero tiene mayor relación el nivel educativo. Es decir, si la persona tiene una avanzada edad, pero al mismo tiempo tiene un nivel de formación alto, no es tan evidente la relación.

Por otra parte, si bien el porcentaje que representan los arrendatarios podría considerarse muy bajo si se compara con los propietarios, es interesante mostrar que dentro de este pequeño grupo, 11 dentro del total de encuestados, el 36,4\%, tiene asistencia técnica permanente y en esa misma proporción posee en sus fincas pasturas mejoradas. El 29,3\% de los arrendatarios tiene pasturas naturales y en esa misma proporción no tienen asesoría técnica permanente; un $9,1 \%$ de los que tienen pastos mejorados tiene asesor para la sanidad animal de manera permanente. De acuerdo con estos datos, se podría inferir que la renta de tierras ganaderas tiende a mejorar la modernización del sector, dado que la finca debe ser productiva y generar recursos para poder ser sostenible económicamente.

\section{Conclusiones}

El nivel educativo es una característica importante debido a la gran influencia que tiene sobre los productores a la hora de modernizar sus fincas. El porcentaje de ganaderos con nivel educativo de solo pri- 
maria es alto; representa, en algunas zonas, el $71 \%$ entre todos los productores. Esta variable se relacionó con la implementación de algunos procesos tecnológicos; se encontró que es determinante, ya que los ganaderos de menor nivel educativo son, al mismo tiempo, quienes no contratan asistencia técnica para atender la salud de los animales. Por su parte, los ganaderos que tienen nivel educativo alto implementan algunos procesos tecnológicos, como contratar asesoría técnica, utilizan pastos mejorados y se ubican de manera estratégica en las zonas del municipio que les brindan mayores ventajas para la producción y comercialización de su ganado.

Otro problema que se evidencia es el envejecimiento progresivo de los ganaderos, lo cual genera preocupación por las implicaciones que tiene este hecho sobre las actividades productivas, entre ellas, la afectación a la modernización tecnológica, generando mayor atraso en el sector y la disminución de la capacidad productiva.

En relación con la tenencia de la tierra, se encontró que la inmensa mayoría de productores son propietarios de sus predios y una minoría trabaja con fincas arrendadas.
Se determina, por tanto, que en estas fincas se tiene presencia de elementos modernizadores, lo cual permite suponer que la renta puede contribuir a la implementación de tecnologías para que la actividad aporte mayores rendimientos.

La actividad ganadera se desarro1la, mayoritariamente, en predios menores de 50 hectáreas, considerados pequeña propiedad; predominan, con los más altos porcentajes en 6 de las 9 zonas. Solo en las unidades 2 y 5 , la mayor proporción se encuentra en el rango de 50 - 500 hectáreas y en la unidad 9, en donde el $100 \%$ de los predios tiene una extensión menor de 50 hectáreas, por lo que puede evidenciarse una mayor desconcentración de la tierra en las zonas donde se tiene mayor valor productivo. A su vez, la mayoría de los predios tiende a combinar la ganadería con otras actividades, especialmente, la agricultura.

La producción ganadera del municipio de Montería se caracteriza por un amplio predominio de los hombres, como protagonistas de esta actividad, representando el 80,1\% de los propietarios; la participación femenina es de solo 16,5\%. La presencia de porcentajes significativos 
(entre el $21 \%$ y el $33 \%$ ) de mujeres más cercanas a la ciudad y en donganaderas se encuentra relaciona- de se da mejor acceso vial. Por el da con la distancia a la cabecera, contrario, el mayor porcentaje de es decir, las zonas donde se halla hombres se encuentra en las zonas más mujeres propietarias son las más alejadas.

\section{Referencias}

Alcaldía de Montería (2002). POT - Plan de Ordenamiento Territorial municipio de Montería 2002-2015. Recuperado de: http://cdim.esap.edu.co/BancoMedios/Documentos\%20PDF/ monteriacordobapot2002-2015-2.pdf

Arias, J. H. (1997). La ganadería en la formación social colombiana: Entre el atraso y la competitividad. Colombia: Ministerio de Agricultura.

Banco Mundial. (2007). Informe sobre el desarrollo mundial 2008. Agricultura para el desarrollo. Washington, DC. Recuperado de: http://siteresources.worldbank.org/ INTWDRS/Resources/477365-1327599046334/8394679-1327614067045/FINAL_WDROV-Spanish-text_9.26.07.pdf

Banco Mundial. (2010). Mujeres tienen menos probabilidades que hombres de participar en el mercado laboral en la mayoría de los países. Recuperado de: http://datos.bancomundial. org/noticias/mujeres_tienen_menos_probabilidades_que_hombres_de_participar_en_el_ mercado_laboral_en_la_mayoria_de_los_paises.

DANE - Departamento Administrativo Nacional de Estadistica. (2010). Encuesta Nacional Agropecuaria. Recuperado de: http://formularios.dane.gov.co/Anda_4_1/index.php/ catalog/25

Estrada, J. (2002). Pastos y forrajes para el trópico colombiano. Manizales: Editorial Universidad de Caldas.

FAO. (2010). Gender and Land Rights-Understanding Complexities; Adjusting Policies. Economic and Social Development, 8. Recuperado de: http://www.fao.org/docrep/012/ al059e/a1059e00.pdf

FAO. (2011). El estado mundial de la agricultura y la alimentación. Roma (Italia). Recuperado de: http://www.fao.org/docrep/013/i2050s/i2050s00.htm

Hagerstrand, T. (1967). Innovation diffusion as a spatial process (Allan Pred, trad.) Chicago: University of Chicago Press. (Obra original publicada en 1953). 
Holguín, V. A., Ibrahim, M., \& Ricardo, M. D. J. (2008). Caracterización de fincas ganaderas de doble propósito en la zona del Pacífico Central de Costa Rica. Revista Colombiana de Ciencia Animal, 1(1), 28-37.

Kristjanson, P., Waters-Bayer, A., Johnson, N., Tipilda, A., Baltenweck, I., Grace, D., \& MacMillan, S. (2010). Livestock and women's livelihoods: A review of the recent evidence. Discussion Paper, 20. Recuperado de: https://cgspace.cgiar.org/bitstream/ handle/10568/3017/Discussion_Paper20.pdf?sequence

Machado, A. (2011). Colombia Rural: Razones para la esperanza. Informe de Desarrollo Humano, PNUD, Colombia. Recuperado de: http://pnudcolombia. org/indh2011/pdf/ informe_completo_indh2011pdf.

Malaquín, I. (2009). Ganadería a tiempo parcial y pluriactividad de la unidad familiar. En H. Morales Grosskopf \& F. Dieguez Cameroni (Eds.), Familias y campo, rescatando estrategias de adaptación. Instituto Plan Agropecuario (pp. 41-47). Uruguay: Instituto Plan Agropecuario.

Marín, C. E. (1996). Sistema de explotación ganadera: notas en torno a su concepto. Lurralde: Investigación y espacio, (19), 89-104.

Montaña, D. F. (2002). Tierra, poder político y reformas agraria y rural. Cuadernos de Tierra y Justicia, 4, 1-48.

Morantes, M.; Dios-Palomares, R.; Peña, M. E.; Rivas, J.; Angón, E.; Perea, J. \& GarcíaMartínez, A. (2014). Incidencia de las características del ganadero en su labor gerencial: un estudio en los sistemas de producción con ovinos de leche en Castilla - La Mancha, España. Revista Cientifica, XXIV(3), 224-232.

Njuki, J., \& Sanginga, P. C. (Eds.). (2013). Women, livestock ownership and markets: Bridging the gender gap in eastern and southern Africa. Londres: Routledge.

Rodgers, E. M. (2010). Diffusion of innovations. Nueva York: The Free Press.

Salgado, C., \& Tréllez, E. (2009). Políticas, estrategias y planes regionales, subregionales y nacionales en educación para el desarrollo sostenible y la educación ambiental en América Latina y el Caribe. OREALC UNESCO. Recuperado de: http://unesdoc.unesco.org/ images/0018/001819/181906S.pdf

Serrano, E., \& Mantecón, Á. R. (2003). Bases para un desarrollo ganadero sostenible: la consideración de la producción animal desde una perspectiva sistémica y el estudio de la diversidad de las explotaciones. Revista española de estudios agrosociales y pesqueros, 199, 159-191.

Tulla, A. F. (1983). El modelo de difusión de T. Hagerstrand. Una aplicación a la ganadería del Pirineo Catalán. Documents d'Analisi Geografica, 2. 
Velasco-Fuenmayor, J., Ortega-Soto, L., Sánchez-Camarillo, E., \& Urdaneta, F. (2009). Factores que influyen sobre el nivel tecnológico presente en las fincas ganaderas de doble propósito localizadas en el estado Zulia, Venezuela. Revista Cientifica, 19(2), 187-195.

Vilaboa-Arroniz, J., Díaz-Rivera, P., Ruiz-Rosado, O., Platas-Rosado, D. E., González-Muñoz, S. \& Juárez-Lagunes, F. (2009). Caracterización socioeconómica y tecnológica de los agroecosistemas con bovinos de doble propósito de la región del Papaloapan, Veracruz, México. Tropical and Subtropical Agroecosystems, 10(1), 53-62.

Wozniak, G. D. (1984). The Adoption of Interrelated Innovations: A Human Capital Approach. The Review of Economics and Statistics, 66(1) 70-79.

Recepción: 5 de enero de 2015

Evaluación: 27 de marzo de 2015

Aceptación: 26 de mayo de 2015 